\title{
Breast Disease is now a Specialist Discipline
}

\author{
Roger W Blamey \\ Nottingham City Hospital, UK
}

Specialisation has always been a part of the evolution of medical practice, initially dividing into the old disciplines of medicine, surgery, and midwifery. Within general surgery my first chief practised what we now recognise as upper GI, urological, thoracic, orthopaedic, cardiac, vascular, plastic, breast, endocrine, trauma and neurosurgery; disasters were frequent, not all attributable to the surgeon for there were equally no specialist radiologists, pathologists nor anaesthetists. We now have super-specialisation in which disease defined disciplines have replaced the old divisions.

In breast cancer in the 1950s the knowledge base was almost non-existent. The treatment was radical mastectomy and irradiation, as it had been for 50 years. So bad were the side-effects and so low the cure rate [1] that many women would have been better left alone, especially those who did not have breast cancer! From the 1970s knowledge and technology have expanded greatly in all disciplines including laboratory science (oestrogen receptor) and pharmacological research (tamoxifen). As changes in practice consequent on this took place there was a marked fall in mortality from breast cancer and case survival of early breast cancer rose from around $55 \%$ at 10 years in the 1970 s to $77 \%$. Two large studies in the UK found better survival correlated with the number of breast cancers operated on annually by the surgeon.

In every discipline there are examples of the great experience gained in specialist working. In breast conserving surgery initially local recurrence rates were high but as specialist units analysed their figures the underlying reasons were recognised and new protocols introduced. To bring this about required an adequate database and multidisciplinary specialist working. Specialist pathologists have introduced immunocytochemistry and other techniques. Radiotherapy has greatly improved and specialist units are introducing new techniques. New therapeutic agents require specialist knowledge from oncologists. Advice on familial risk comes from close cooper- ation between the geneticist, surgeon and radiologist. The quality of life of women with breast cancer has improved with the introduction of specialist support staff and cosmetic surgical techniques by specialist breast surgeons have played a part. Specialist care has improved all facets of disease management. The knowledge base required and the mastery of techniques in each discipline and the rate of change of these, mean that no-one can remain competent outside specialist multidisciplinary working.

Implementation of specialisation has varied from country to country and is probably most advanced in the UK. The British Association of Surgical Oncology Breast Group in 1991 advocated team working by specialists. These views were unwelcome to many general surgeons who preferred a wide working base, but the view of the patients must be paramount and they want expertise. However the difficulties of managing screen detected lesions, techniques in diagnosis and pathology, new agents and (regrettably) the increase in medical litigation, led to the collapse of resistance and now almost all cases of breast cancer in the UK are diagnosed and managed by teams of specialists working in Breast Units.

EUSOMA (European Association of Breast Specialists) published guidelines 'The Requirements of a Specialist Breast Unit' [2]. These describe in detail specialist multidisciplinary working in all disciplines within a single integrated unit, set out as a series of quality objectives alongside outcome measures. Units must offer a total breast service and require a sufficient caseload to justify expense and maintain expertise. The structure is of a core team of specialists in surgery, pathology, radiology, diagnostic radiation and medical oncology as well as patient support. Backup is given by recognised individuals in a non-core team from associated disciplines (genetics, plastic surgery, psychiatry, physiotherapy and palliation).

There was considerable interest and a number of claims arose to satisfy the 'Requirements' when they clearly did not do so.

\begin{tabular}{ll}
\hline KARGER & ๑ 2006 S. Karger GmbH, Freiburg \\
Fax +49 7614520714 & Accessible online at: \\
$\begin{array}{l}\text { E-mail Information@Karger.de } \\
\text { www.karger.com }\end{array}$ & www.karger.com/brc
\end{tabular}

Prof. Roger W Blamey, MD 
To overcome this a process of accreditation was established by EUSOMA. Certification of screening programmes is available from EUREF (European Reference Group for Breast Cancer Screening). In most countries screening programmes are independent of breast units and the EUSOMA and EUREF processes are therefore separate but share the view that they should be designed and run by specialists in the field.

Details of the process and the procedure for individual units were described, amended and finally published [3]: on application a unit completes a questionnaire based on the guidelines [4] and is then visited by an accrediting group of four international specialists (surgeon, radiologist, pathologist and breast care nurse). Consequent on this a report is written by the Accreditation Coordinator (R.W.B.) and considered by the Accreditation Board.

This first assessment, for which 'Initial Accreditation' may be given, is of the capacity of a unit to fulfil the guidelines [4] The essential criteria which a unit must satisfy are a sufficient case load, a single integrated unit, specialists in all disciplines, multidisciplinary working, covering all aspects of breast disease, good service provision, good patient support, data collection and audit. 'Full Accreditation' is based on outcome measures and performance indicators and requires 5 years of data and will be largely electronic; 're-accreditation' every subsequent 5 years will be similar. Data collection is being established on a modification of the QT database [5].

Accreditation began slowly and procedures became well defined and smooth. Over the last 6-month period units have been visited at the rate of 2 per month; overall 17 units have been accredited of which a number are well known interna- tionally and a further 3 visited; 10 await visits. An important achievement is that all units have been given recommendations on areas which were unsatisfactory and without exception all units have been keen to comply, even with minor, nonmandatory points. Most breast cancer research has come from large integrated breast units and these units also hold continuing education courses for trainees and specialists and have a strong student teaching commitment.

In conclusion it is now well recognised that breast cancer should be managed by breast specialists working in breast units. The EUSOMA Requirements [4] are well accepted for defining the breast unit, the team and its working. To ensure compliance Accreditation has been established by EUSOMA; the process is voluntarily but as it becomes more and more accepted by doctors and patients all units will wish to show themselves as accreditable. This will result in an excellent structure for breast disease, to which all European citizens will have access.

\section{References}

1 Brinkley D, Haybittle JL: Long-term survival of women with breast cancer. Lancet 1984;1:1118.

2 Blamey RW and EUSOMA writing party: The requirements of a specialist breast unit. Europ J Cancer 2000;36:2288-2293.

3 Blamey RW, Cataliotti L: EUSOMA accreditation of breast units. Europ J Cancer 2006, in press.

4 Blamey RW, Cataliotti L: The requirements of a specialist breast unit, first revision; in: European Guidelines for Quality Assurance in Breast Screening and Diagnosis (ed 4). European Community, 2006, pp 343-354.

5 Ponti A, Segnam N, Blamey RW: Data collection on treatment of screen detected lesions; in: European Guidelines for Quality Assurance in Breast Screening and Diagnosis (ed 3). European Community, 2002, pp 221-237. 